\title{
Assessment of a Heterogeneous Environment
}

\author{
Alexander Zverovshchikov \\ Department of Technology and Equipment of Mechanical Engineering, Penza State University, \\ 40 Krasnaya Street, Penza 440026, Russian Federation
}

azwer@mail.ru

Keywords: Heterogeneous Environments, Cutting, Coating, Metal, Technologies

\begin{abstract}
An approach to the assessment of a heterogeneous environment in accordance with the proposed concept of a heterogeneous environment is described. The classification of industrial heterogeneous media by technological feature is proposed. The processes described by the proposed approach include the process of metal cutting, surface plastic deformation, chemicalthermal treatment, formation of coatings, welding, most of the processes that change the level and gradient of hardness and internal stresses in the surface layer of the material. Application of the proposed principles makes it possible to find new directions in the study of such processes.
\end{abstract}

\section{Introduction}

The concept of heterogeneous environments is used in most scientific and technical branches of knowledge to describe a wide variety of systems. The basic concepts of a heterogeneous system or environment are described in works on physics, chemistry, sociology, economics, and biology $[1,2]$. The main features of a heterogeneous environment are the presence of areas with different characteristics of the state and boundaries between them [3].

There are a number of particular definitions of heterogeneous systems in materials science, physics, which are reduced to macroscopic thermodynamic inhomogeneity, differences in physical properties, chemical composition of parts (phases) [4], electrical properties [5]. In the definitions, in a number of special cases, there is a connection with dispersed systems, determined by the degree of development of the interfaces between the constituent phases [6]. A very large number of studies are devoted to the kinetics of heterogeneous media in chemistry, dynamics in mechanics $[7,8,9,10]$, in particular in the study of the physics of combustion and explosion $[11,12,13,14,15]$.

Attempts to create generalized universal models for describing and calculating heterogeneous media have been made repeatedly in various fields of knowledge [16].

The aim of this work is an attempt to generalize and localize the concept of a heterogeneous environment from the point of view of industrial technologies. The urgency of the problem is substantiated by the need for a systematic approach in the analysis of behavior and the creation of heterogeneous industrial environments.

\section{Characteristics of Technological and Industrial Heterogeneous Environments}

Let's highlight the main characteristics of technological and industrial heterogeneous environments as a basis for their classification. Considering a heterogeneous environment within the framework of the classical approach [17], we note that it is a system of elements separated by boundaries. The external boundaries of the system that define the area of analysis will be considered technical constraints depending on the production problem. Let's define the main characteristics that determine the difference between the elements of a heterogeneous technical environment:

- Mechanical characteristics: hardness, elasticity, viscosity, optical permeability, etc .;

- Geometric characteristics;

- Electromagnetic characteristics. 
Along with the above, it is advisable to introduce the concept of the spatial dimension of the medium. So, one-dimensional environments can be considered only in some contradiction with the term "environment" itself, which presupposes the environment, the scope of an object, in fact, presupposes multidimensionality. However, the consideration of such objects as, for example, an extended fiber, homogeneous in properties in the cross section, but variable in properties along the length, which is several orders of magnitude larger than the cross section size, allows one to consider a fiber, a thin conductor, and similar objects as a one-dimensional heterogeneous medium.

Two- and three-dimensional heterogeneous media are widespread in industrial engineering and technology. Examples include filter media, rough or abrasive surfaces, free abrasive media, construction and tool materials, and many other technical objects.

Considering the geometric characteristics of the elements of the medium, as the simplest, we can conclude that the characteristic of heterogeneity, even in the size of particles, when examined in detail, can be quite complex. For example, the medium of an abrasive powder with a single fraction differs significantly from a medium with a multifraction composition. Thus, we can talk about the presence of a second order or a level of heterogeneity. This, in turn, leads to the need to introduce a separation criterion according to the corresponding indicator for particles of the medium, and in some cases, its zones and regions. So, for abrasive media, the range of sizes of fractions is stipulated by the standards [18].

However, even in this case, the characterization of the particles will be incomplete, since the existing methods do not take into account the shape of the grain, which can radically differ within one fraction and, accordingly, affect the properties of the heterogeneous medium (for example, by changing the coefficients of internal friction).

Using this simplest example, one can hypothesize that the heterogeneity in each of the above groups of characteristics can be described by a multifactorial vector

$$
Q_{i}=\left\{\begin{array}{l}
X_{1} \\
X_{2} \\
X_{i} \mathrm{x}, \\
\cdots \cdots \\
X_{n}
\end{array}\right.
$$

where $X_{i}$ - a characteristic of an element of the environment. Moreover, the dimension of the vector will be determined both by the boundaries of the system under consideration and by the significance of the characteristic for the theoretical or applied problem being solved.

The level or order of heterogeneity may or may not be relevant when modeling the behavior of the environment at different boundaries and purposes of analyzing the system under study. Obviously, for the vector (1), an additional dimension is added, which complicates the study.

Let us further consider such an indispensable element of a heterogeneous environment as the boundary between elements with different properties. The boundary is characterized by a change in the properties of the environment, which must be assessed by quantitative criteria. In this case, even speaking about the spatial geometric boundaries of a heterogeneous medium, one can operate with such a characteristic or concept as the thickness of the boundary. For example, a heterogeneous medium in the form of an emulsion or suspension has clearly defined boundaries of elements at the atomic level. Solid solutions of metals, in which the elemental components can be clearly distinguished, have more diffuse boundaries due to the diffusion of materials.

Thus, if neighboring elements of the environment have differences in the $X_{\mathrm{i}}$ parameter of the heterogeneous environment, then the boundaries will be determined by the criterion difference 


$$
\Delta_{i}=X_{i}^{\prime}-X_{i}^{\prime \prime}>\Delta k,
$$

where $\Delta k$ - the difference between the element separation parameter, which is critical for the problem under consideration.

Thus, through $\Delta k$, it is possible to quantitatively determine the size (thickness) of the boundary, for example, zones of electrolyte solution of different concentrations, which form a heterogeneous medium according to the mentioned parameter. In addition, criterion (2) is inevitably associated with the spatial gradient $\frac{d X_{i}}{d L}$ of the considered parameter in the dimension of the space $\mathrm{L}$, which allows us to introduce the concept of border contrast. Indirectly, criterion (2) allows one to characterize the level of interaction between elements of a heterogeneous environment.

In a number of cases, such an approach requires certain assumptions when assessing the boundaries of the components of a heterogeneous medium (let's call them monoelements). One of the striking examples of a high-contrast boundary, which, meanwhile, has a certain volume and gradient of the components of a heterogeneous medium is the boundary of a galvanic or other coating on the base material (Fig. 1).

On the one hand, the boundary between coating and base materials has a clear contrast in terms of chemical and structural composition. However, almost any surface has a complex of microroughnesses. If we take into account such a roughness indicator as the length of the supporting surface tp, the mechanical properties of the coating layer at a thickness corresponding to the height of the microroughness of the surface of the base material $R_{\max }$ will differ significantly. Thus, from the point of view of physical and mechanical properties, the boundary will be blurred and have a sufficiently large thickness, and this thickness, as well as the gradient of properties determined by the parameters $R_{\max } t_{p}$.

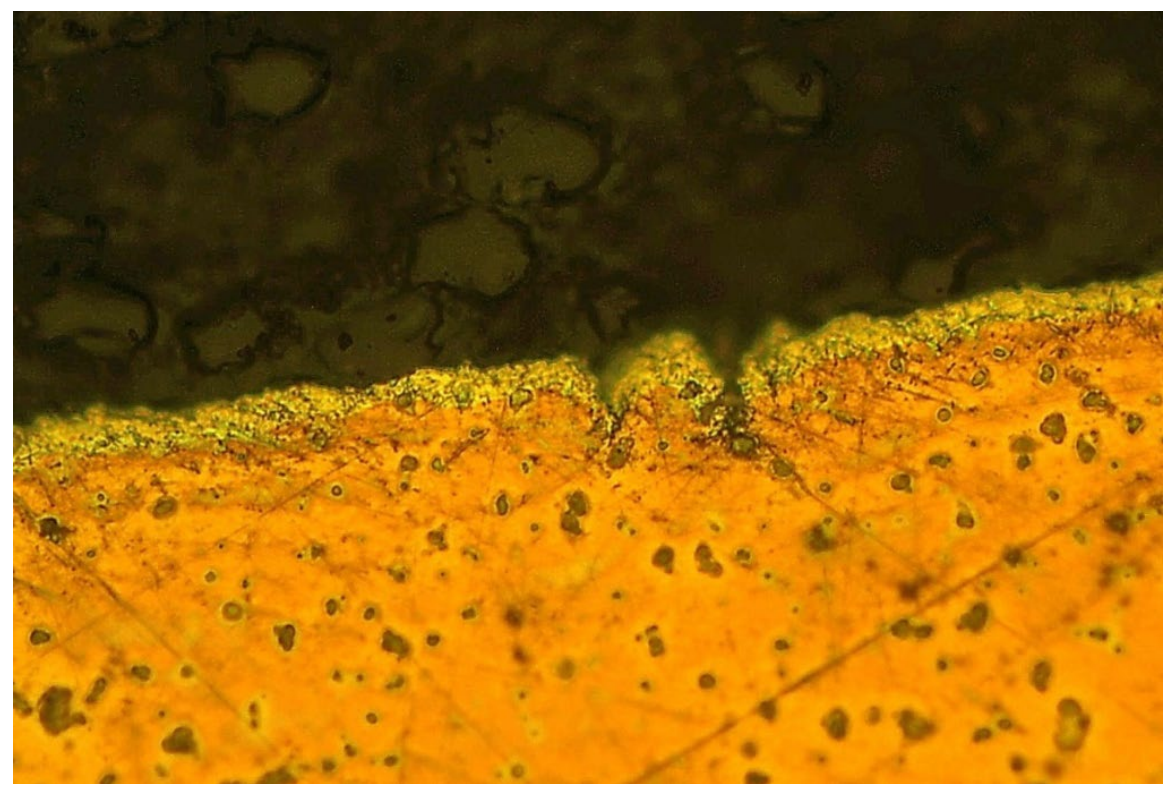

Fig. 1. Boundary of a metal coating on a metal base

When comparing the matrix of vectors $Q_{1}$ and $Q_{2}$ for different phases, it is possible to obtain a situation when the boundary characterized by the parameter $X_{c}$ does not coincide with the boundary by the parameter $X_{n}$

Or the boundary may be characterized by a critical change in only one parameter. For example, for electroplating, the presence of a certain concentration of microcracks and its change within (2) 
over the surface of the part indicates the heterogeneity of the coating on the surface, and all other indicators can be unchanged.

Thus, the localization of the border, depending on the tasks of research or modeling, can be formulated by one or more parameters of the vector (1), have a different gradient depending on the objectively present or subjectively specified criterion (2).

When considering the boundaries between the elements, one more key characteristic of the heterogeneity of the considered environment can be distinguished. This characteristic can be the length of the boundaries per unit of the dimensional characteristics of the medium. Taking into account the dimension of the medium, we can talk about point, linear, volumetric boundaries, as well as boundaries with a higher dimension. For example, the boundary between metals, in a simplified form, is considered as a certain line between grains in microscopic examination of a thin section. In reality, firstly, such a boundary is characterized by a certain criterion difference $\Delta k$, determined, as mentioned above, by the degree of diffusion of materials, and secondly, it is a complex three-dimensional surface. Then, the dimensional characteristic of the boundary will be the volume $V_{r}$, determined by the expression

$$
V_{r}=S_{r} * \Delta k \text {. }
$$

Accordingly, a characteristic of the heterogeneity of such an environment can be considered the indicator

$$
Q=\frac{V_{r}}{V_{a}} \leq Q_{k},
$$

where $V_{a}$ is the unit of the considered dimensional characteristic of a heterogeneous medium, for example, volume; $\mathrm{Q}_{k}$ is the critical ratio of the total dimensional characteristics of the boundaries and the total investigated volume for the problem under consideration.

The volume $V_{a}$ for the case under consideration includes the components that can be considered homogeneous media for a particular study.

The considered case is a particular consequence of the proposed hypothesis for geometric threedimensional boundaries.

Characteristics (3) and (4) are especially relevant precisely for geometric boundaries, since they make it possible to take into account the state of the surface and the development of the surface profile with the same dimensions of the elements of the components of the heterogeneous medium. In particular, for powder metallurgy, it is this indicator that will affect the degree of particle activation. For abrasive media with the same particle size - their surface area will determine the aggressiveness and abrasive ability during cutting,

Using the above approach, almost the majority of technical objects can be classified as heterogeneous media: structured materials, coatings, processing media, raw materials, materials obtained by using additive technologies. Even materials that are technically considered monolithic, for example, hypoeutectoid alloys of the iron-carbon diagram, can be classified as heterogeneous.

However, simplifying vector (1) i.e. removing from the vector (1) the corresponding indicators in the analysis of such materials and considering it conditionally monolithic, taking out of the analysis of the system the features of the structure and structure, the process of dispersing the material, for example, by the processes of abrasive or blade cutting, can be considered the synthesis of a heterogeneous structure from a homogeneous medium.

The above hypothesis makes it possible to form an almost universal approach to the study and modeling of a variety of processes associated with the formation of heterogeneous environments. For the technology of mechanical engineering, one of the basic ones is the processes of blade or 
abrasive cutting of metals and alloys. One way or another, but the essence of these processes is reduced to the dispersion of a monolithic solid material.

The process of dispersing a monolithic (conditionally monolithic) solid material can be considered as an increase in the degree of heterogeneity of the initial medium. The formation of cracks during destruction or dispersion is accompanied by the formation of interfaces and spaces between them. The presence of an initial crack - fulfills condition (2), at least the geometric characteristics of the formed elements appear in the vector (1). With an increase in the heterogeneity of the medium under consideration, its properties change dramatically.

If we consider various cutting methods and compare them according to the results of dispersion, then the resulting chips, as a heterogeneous medium, can be quantitatively characterized by the indicator (3). In this case, the increase in the surface of the boundaries of elements can be described by the function

$$
Q=f(A, G, P, T, N) \text {, }
$$

where $T$ is the cutting time, $N$ is the power consumed by the process, $G$ is the geometry parameters of the cutting wedge, $A$ is the cutting modes, $P$ is the complex of physical and mechanical properties of the material being processed.

In accordance with the theory of brittle fracture [19], the considered increase in heterogeneity in the form of processing a solid material into shavings requires energy consumption, which is determined by the properties of its own material and the characteristics of the elements. Thus, reducing the size of the elements requires higher energy consumption for dispersion, which does not contradict the known provisions of the theory of cutting.

If we generalize the influence of the dimensional characteristics of the boundaries and, accordingly, the parameter (5) on energy costs, then it will obviously be nonlinear, and the nonlinearity, depending on the parameter (4), will be changing (Fig. 2).

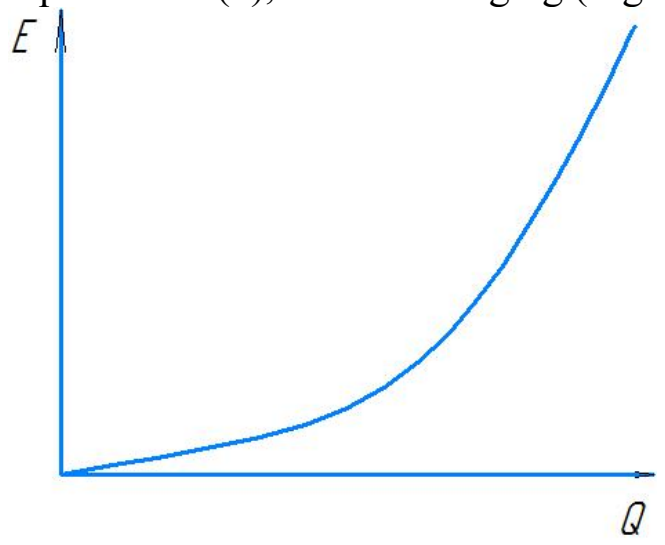

Fig. 2. Influence of the degree of development of the boundaries between the components of a heterogeneous environment on the energy consumption of its formation

If we consider the processes of electroplating as the transformation of a two-component medium base-electrolyte into a three-component base-coating-electrolyte, then an increase in heterogeneity is also associated with the formation of another interface and with significant energy consumption.

There are known processes associated with the joining of metal surfaces by explosion welding [20]. In this case, the disappearance of two metal-gas-metal boundaries and the formation of a metal-metal boundary. Although the number of boundaries decreases, the new boundary is characterized by a sharp increase in volume due to the diffusion of metals. Thus, the heterogeneity 
of the environment in terms of indicator (3) increases, a spatial gradient $\frac{d X_{i}}{d L}$ of the boundary appears, respectively, this requires significant energy consumption, which is technologically confirmed.

A similar situation arises in the manufacture of suspensions, emulsions and other heterogeneous media consisting of substances in different phases.

\section{Conclusion}

Summarizing, we can put forward a hypothesis that increasing the heterogeneity of the environment requires energy costs and reduces the level of entropy.

The proposed hypothesis will obviously have many boundary conditions, for example, dispersion at the molecular level, processes related to nuclear interactions, in many cases may contradict the proposed hypothesis.

However, the processes described by the proposed approach include the process of metal cutting, surface plastic deformation, chemical-thermal treatment, formation of coatings, welding, most of the processes that change the level and gradient of hardness and internal stresses in the surface layer of the material. Application of the proposed principles makes it possible to find new directions in the study of such processes.

\section{Acknowledgments}

The work was carried out within the framework of the project No. 0748-2020-0013 "Scientific principles of the formation of heterogeneous structures by methods of physical and chemical dispersion" (state assignment to the university in the field of scientific activity. Customer: Ministry of Science and Higher Education of the Russian Federation).

\section{References}

[1] K Shannon, Mathematical theory of communication. «Works on Information Theory and Cybernetics, Moscow, Publishing of Foreign Literature Publishing House (1963) pp.243-332.

[2] B.A. Erznkyan, Technological and institutional development of the socio-economic system in a heterogeneous environment, JOURNAL OF INSTITUTIONAL STUDIES, Volume 4, No. 3. 2012.

[3] V.S. Igropulo, Heterogeneous environment with active components: physical system, mathematical model, real prototypes. Proceedings of Bulletin of Stavropol State University 38/2004 pp.113-116.

[4] V.I. Gorodnichenko and others, Materials Science: Workshop, Moscow - Logos Proceedings, 2003.

[5] G.I. Dulnev and V.V. Novikov, Transfer processes in heterogeneous media, Leningrad, Energoatomizdat Publishing, Leningrad Branch, 1991.

[6] Materials Science. Technology of structural materials: textbook. manual ed. B.C. Cherednichenko. - 2nd ed. - Moscow; Omega-L Publishing House, 2006.

[7] L.Barre, Kinetics of Heterogeneous Processes. - Moscow, Mir Publishing House, 1976.

[8] 8 D.A. Frank - Kamenetky, Diffusion and heat transfer in chemical kinetics, Moscow Publishing House Science, 1987.

[9] V.F. Kuropatenko, New models of continuum mechanics Engineering Physics Journal., 2011, Volume 84, No. 1. p. 74-92. https://doi.org/10.1007/s10891-011-0457-0 
[10] V.F. Kuropatenko, Model of a multicomponent environment, Reports of the Academy of Sciences, 2005, Volume 403, No. 6. - p. 761-763

[11] V.Fortov, Powerful shock waves and extreme states of matter. Moscow, Bukos Publishing House, 2005. https://doi.org/10.1238/Physica.Topical.116a00007

[12] A.P. Aldushin, T.M. Martemyanova, A.G. Merzhanov, B.I. Khaikin and Shkadinsky K.G. Self-Oscillatory Propagation of a Combustion Front in Heterogeneous Condensed Matter, Journal of Combustion and Explosion Physics. 1973. Vol. 9, No. 5. p. 613-626. https://doi.org/10.1007/BF00742879

[13] V.G .Prokofiev and V.K. Smolyakov, Nonstationary regimes of combustion of a binary gasless mixture when ignited by a heated wall, Journal of Combustion and Explosion Physics. 2005.Vol. 41, No. 2. pp. 45-50. https://doi.org/10.1007/s10573-005-0018-y

[14] K.A. Aligozhin and A.G. Knyazeva, Modeling of transformation modes that are realized when joining dissimilar materials using solid-phase synthesis, Journal of Mathematical Modeling in Natural Sciences. 2013. No. 1. pp. 8-10

[15] A.Yu. Krainov and DS Shultz, Mathematical modeling of an ultrahigh-speed process in heterogeneous reacting powder mixtures, Journal of Computer Research and Modeling, 2011, Volume 3, No. 2, pp. 147-153. https://doi.org/10.20537/2076-7633-2011-3-2-147-153

[16] V.E. Fortov, Equations of state of matter: from ideal gas to quark-gluon plasma, Moscow, Fizmatlit Publishing House, 2012.

[17] R. I. Nigmatulin, Fundamentals of mechanics of heterogeneous media, Moscow, Science Publishing House. 1973.

[18] GOST R 52381-2005 Abrasive materials grain size and grain size composition of grinding powders. Grain composition control.

[19] V. M. Markochev and M. I. Alymov, On the theory of brittle fracture by J. Frenkel and A. Griffiths, CHEBYSHEVSKY Sbornik, 2017 Volume 18 Issue 3. https://doi.org/10.22405/22268383-2017-18-3-381-393

[20] Vladimir A. Grachev, Andrey E. Rozen, Yury P. Perelygin, Sergey Yu. Kireev, Irina S. Los Multilayer corrosion-resistant material based on iron-carbon alloys// Heliyon 6 (2020) e04039. https://doi.org/10.1016/j.heliyon.2020.e04039 